\title{
Validación y verificación de métodos de ensayos. Un dilema en los laboratorios de ensayos y en las auditorías de la acreditación
}

\section{Gustavo Delgado ${ }^{1^{*}}$}

1. Universidad Nacional Autónoma de Nicaragua, León (UNAN-LEÓN). Facultad de Ciencias y Tecnología, Departamento de Química, Laboratorio de Análisis de Trazas de Metales Pesados (LATMP), Edificio de Ciencias Básicas, León, Nicaragua. Tel: (505) 2311 5013, ext: 1132. Fax: (505) 23114012.

\section{RESUMEN}

El presente artículo tiene como objetivo fundamental establecer en forma clara y sencilla la diferencia entre validación y verificación de un método de ensayo. Se presenta una breve discusión de la selección y validación de los métodos de ensayos establecidos en la norma ISO/ IEC 17025:2005. Se describe el proceso de normalización de un método. Se describen brevemente los parámetros de la validación como indicadores característicos del desempeño del método, brindando algunas breves orientaciones para su aplicación. Se presentan algunos contenidos de métodos normalizados, indicando las especificaciones a partir de los datos de validación. Y por último se describe el proceso de verificación y su diferencia con la validación, haciendo énfasis en la confirmación de las especificaciones del método.

Palabras claves: Validación de métodos de ensayos, verificación de métodos de ensayos.

\section{INTRODUCCIÓN}

Desde mediados de los años 90's, la acreditación en los laboratorios de ensayos ha venido tomando gran importancia para la exportación e importación de productos de intercambio comercial. La acreditación nace como una necesidad para favorecer los intercambios comerciales y eliminar los obstáculos técnicos al comercio, a través de la política comercial establecida por los acuerdos del GATT (Acuerdo General sobre Comercio y Aranceles) en los años 70's y en la Ronda del Uruguay en $1994^{[1]}$.

Este concepto fue tomado por el gobierno de Nicaragua en 1996 ante el fenómeno de la globalización de los mercados y los Tratados de Libre Comercio. El Ministerio de Comercio Industria y Comercio (MIFIC) creó la ley $219^{[2]}$ donde se constituye la Comisión Nacional de Normalización Técnica y Calidad, con el fin de coordinar la elaboración y aprobación de normas técnicas obligatorias y voluntarias para la comercialización de productos tanto a nivel nacional como internacional.

Bajo esta política nace la Oficina Nacional de Acreditación $(\mathrm{ONA})^{[3]}$, la cual está dedicada a la acreditación de Organismos Evaluadores de la Conformidad (OEC). Dentro de esta categoría entran los laboratorios analíticos y microbiológicos, y para acreditarse deben tener la competencia técnica basada en la norma NTN 0400105 equivalente a la norma ISO/IEC 17025:2005 ${ }^{[4]}$. En el campo de la salud los laboratorios clínicos se deben regir por la norma ISO 15189:2007[5].
Uno de los principales requisitos que exige la norma ISO/ IEC 17025:2005 (numeral 5.4) es el de utilizar métodos normalizados, los cuales deben estar validados y/o previamente verificados, con el fin de garantizar a los clientes la trazabilidad, precisión y confiabilidad en los resultados. Los laboratorios y los auditores, durante el proceso de acreditación de los laboratorios de ensayos, se enfrentan al problema de establecer la diferencia entre validación y verificación de los métodos de ensayos.

El presente artículo pretende explicar en forma clara y ejemplificada esta diferencia. Para ello, se describe el numeral 5.4 de la norma ISO/IEC 17025:2005 a través del diagrama de espina pescado, en lo referente a los requisitos de selección y validación de métodos de ensayos. Se discute el concepto y los parámetros de la validación de un método analítico. Se presentan los contenidos de diferentes métodos normalizados. Se define el concepto de verificación y su aplicación a las especificaciones de los métodos normalizados y se discute la diferencia entre la verificación y la validación de los métodos de ensayos.

\section{REQUISITO PARA LA SELECCIÓN Y VALIDACIÓN DE MÉTODOS SEGÚN ISO/IEC 17025:2005}

Para garantizar la calidad de los resultados a los clientes, la norma establece que el laboratorio debe utilizar métodos normalizados. Es decir, validados y verificados. En el diagrama de Ishikawa se presenta de manera resumida los componentes de este numeral, figura 1. 


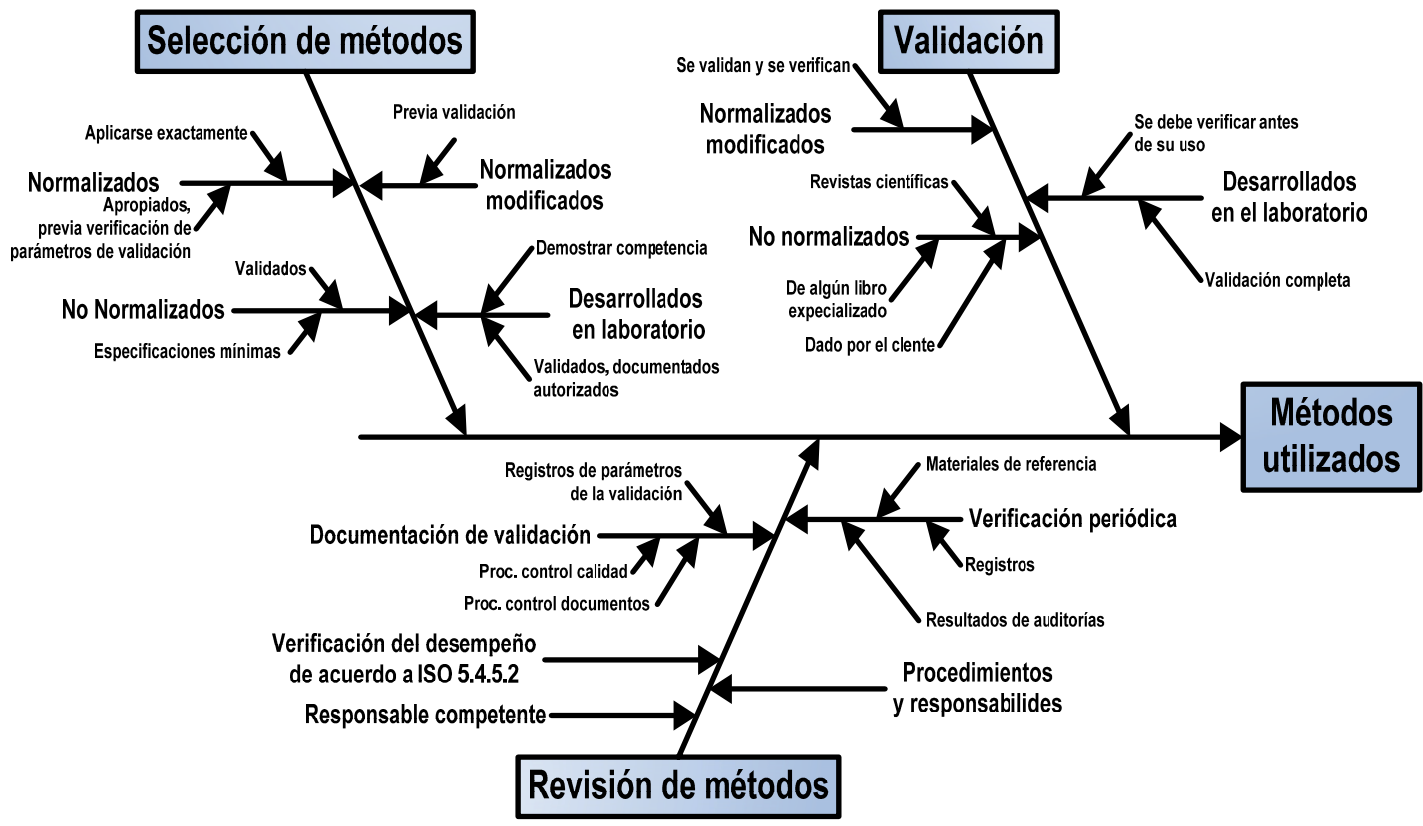

Figura 1. Requisitos para la selección y validación de métodos de ensayos.

El laboratorio debe seleccionar los métodos de acuerdo a los requisitos del cliente, siguiendo las etapas de la evaluación de la conformidad, tales como la selección, determinación y revisión y atestación ${ }^{[6]}$. Un laboratorio acreditado o que tiene implantado el sistema ISO 17025 , debe utilizar métodos normalizados previamente verificados. Si no dispone de estos métodos, puede hacer uso de métodos no normalizados, normalizados modificados o desarrollados en el laboratorio, previa validación que demuestre la aptitud de satisfacer los requisitos del cliente.

La validación consiste en evaluar las características del desempeño del método para demostrar que cumple con esos requisitos. Los métodos no normalizados pueden ser tomados de algún libro especializado, una revista científica o proporcionado por el cliente. Los métodos normalizados modificados son los que han sido modificados en el laboratorio; por ejemplo, si se cambia el tipo de reactivo por otro equivalente, si se preparan volúmenes de patrones o reactivos diferentes a lo establecido en el método normalizado o si se toman cantidades de muestras diferentes, o cualquier otra modificación que no afecte la robustez del método.

Si una modificación afecta la robustez del método, se debe tratar como un nuevo método y se debe demostrar su aptitud para el uso previsto a través de la validación. Los métodos desarrollados en el laboratorio deben ser optimizados y validados.
Es importante, señalar que el laboratorio debe estar en constante revisión de sus métodos. Deben ser verificarlos periódicamente por personal competente, documentarlos y tomar decisiones en el momento apropiado para una revalidación. En la medida de lo posible debe utilizarse métodos actualizados, de acuerdo al avance de la ciencia y la tecnología y a las exigencias de los tratados comerciales con los países importadores.

\section{VALIDACIÓN DE MÉTODOS DE ENSAYOS}

El concepto de validación se define como la confirmación de que los requisitos especificados son adecuados para un uso previsto ${ }^{[7]}$. Es decir que, a través de evidencias objetivas se confirma que los requisitos particulares son apropiados para una aplicación específica. Aplicada a los métodos analíticos se puede definir como el proceso de establecer las características del desempeño y limitaciones de un método y la identificación de las influencias que pueden cambiar estas características ${ }^{[8]}$, demostrando que cumplen con las especificaciones requeridas por el cliente.

Para interpretar este concepto, es necesario identificar los requisitos del cliente. Cuando un cliente requiere los servicios de un laboratorio, el cliente espera resultados de calidad de acuerdo a los indicadores que él tiene preestablecidos, según el tipo o naturaleza de la matriz de la muestra suministrada al laboratorio. Estas matrices pueden ser ambientales, alimenticias, fármacos, biológicas, etc. 


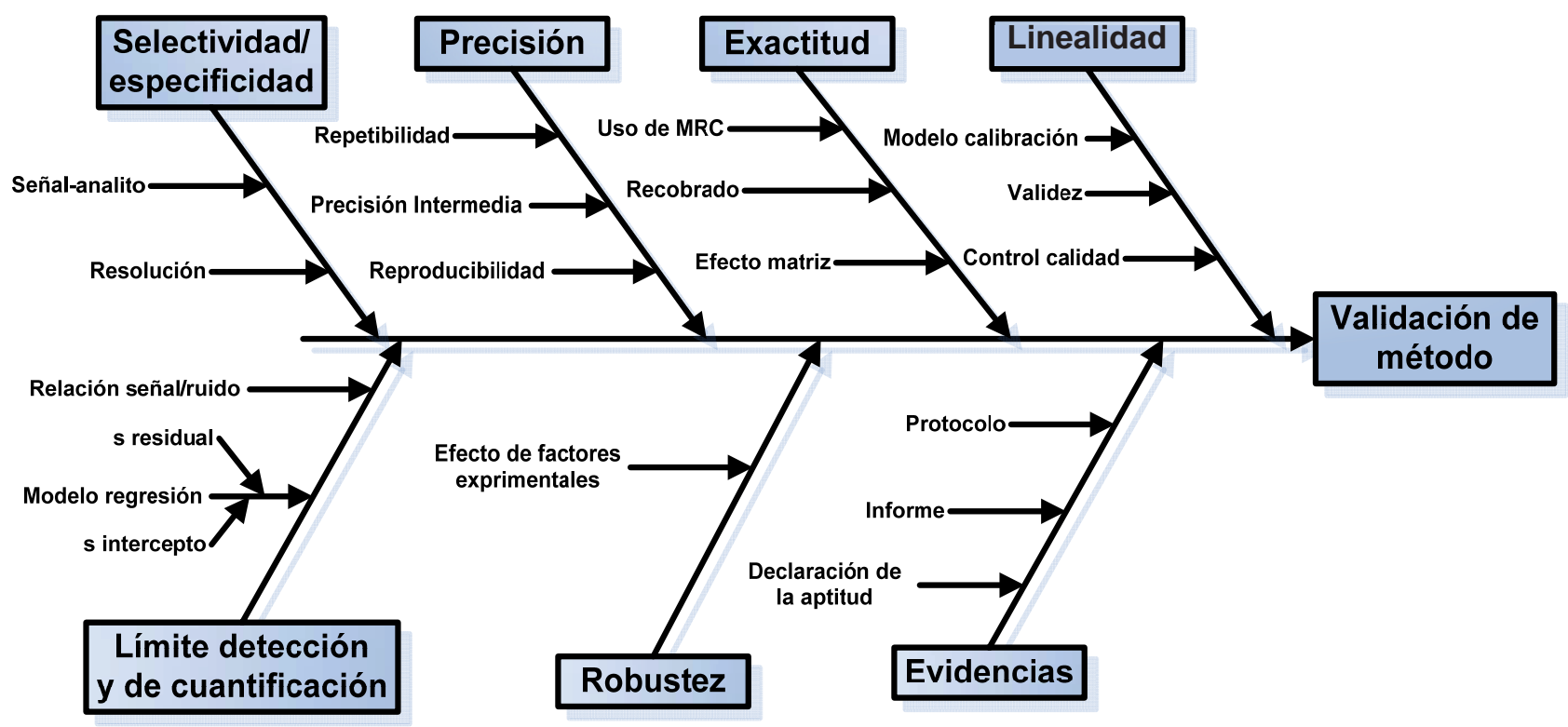

Figura 2. Parámetros de la validación de un método

El cliente espera que las especificaciones de su muestra sean confirmadas correctamente: valores exactos, precisos y confiables. Para que el laboratorio garantice estos resultados debe utilizar métodos validados. Para validar el método es necesario evaluar las características del desempeño del método. De acuerdo a la norma ISO/ IEC 17025:05[4], estas características son: selectividad/ especificidad, precisión, exactitud, linealidad y rango, límite de detección, límite de cuantificación, robustez.

En la figura 2 se presentan los componentes de la validación. La selectividad y especificidad, se refiere a la capacidad que tiene la técnica utilizada para que la señal responda claramente al analito presente en la muestra. Si existen uno o más componente en la muestra que pudieran responder en la zona de respuesta del analito, debe demostrarse buena resolución, de tal manera que se diferencie la señal del analito de los otros componentes.

La exactitud debe evaluarse utilizando materiales de referencia cuyos valores de referencia tengan trazabilidad. El uso de materiales de referencia certificados (MRC) ${ }^{[9]}$ es muy importante para demostrar la trazabilidad de los resultados. Se pueden usar materiales de referencia internos, que han sido preparados en el laboratorio por comparación con los MRC. Si no se disponen de muestras de referencia certificadas, se deben elaborar muestras contaminadas (es decir fortificadas) con patrones de referencia certificados y se calcula el recobrado. Estos materiales también sirven para evaluar las interferencias de la matriz de la muestra y se puede demostrar si hay efecto de matriz (efecto intensificador o depresor) ${ }^{[10]}$.

La precisión se evalúa en tres condiciones: repetibilidad, precisión intermedia y reproducibilidad ${ }^{[19]}$. La repetibilidad puede evaluarse efectuando 10 réplicas por el mismo analista, el mismo instrumento, las mismas condiciones ambientales y locales. Se expresa como desviación estándar $\left(s_{r}\right)$ o como desviación estándar relativa $\left(R S D_{r} \%\right)$ o como coeficiente de variación (CV\%), término poco usado actualmente. La precisión intermedia representa la capacidad de reproducir los resultados de una misma muestra dentro del laboratorio con analistas diferentes, diferentes días, diferentes instrumentos, etc.

Es decir, la precisión intermedia representa las variaciones dentro del laboratorio ${ }^{[12]}$ y se expresa como desviación estándar $\left(s_{t}\right)$, esto significa evaluar la reproducibilidad interna. Por otro lado, es muy importante que el laboratorio participe en ensayos de comparación con otros laboratorios con el fin de evaluar su reproducibilidad. El concepto de reproducibilidad se ha destinado exclusivamente a la variación entre laboratorios y se expresa como desviación estándar $s_{R}$ o $R S D_{R} \%$.

Es importante destacar que, los datos de precisión son tomados en cuenta en el cálculo de la incertidumbre en los análisis de rutina. Este último parámetro no se discute en este artículo, pues es un parámetro propio de un resultado de ensayo; no obstante, algunos consideran 
este parámetro como parte de la validación porque es un indicador más del desempeño del método ${ }^{[12]}$.

La linealidad representa el modelo de respuesta del instrumento en función de la concentración del analito. Se establece el rango de concentraciones donde la señal analítica es proporcional a la concentración. Puede darse el caso de que se tengan 2 zonas de linealidad, una para bajas concentraciones y la otra para altas concentraciones.

Dependiendo del contenido del analito en la muestra se selecciona el rango de linealidad apropiado. La linealidad se evalúa con el coeficiente de determinación $\mathrm{r}^{2}$ entre más se aproxime a la unidad el modelo se ajusta mejor a la proporcionalidad entre la señal analítica y la concentración. La linealidad debe verificase con la gráfica de los errores residuales o por un análisis de varianza que compara el modelo con los residuales. También, puede aplicarse el test de Mande ${ }^{[13]}$ que compara el modelo de la línea recta con el modelo de segundo orden.

El límite de detección (LD) se define como la cantidad mínima de analito en la muestra que se puede detectar. Es importante señalar que lo ideal es evaluar la respuesta de la matriz como blanco. Y luego añadir estándar (analito) a la matriz en concentraciones tales que la razón entre la señal del analito y la de la matrizblanco sea mayor que 3. En la mayor parte de los casos es difícil obtener la matriz-blanco y lo que se hace es evaluar el LD a partir de la desviación estándar residual de la curva de calibración por adición patrón ${ }^{[14]}$.

En estas condiciones la dispersión de los puntos de calibración representa la variabilidad de la respuesta debido al ruido producido por blancos-reactivos, por el instrumento y por el efecto de la matriz. Muchas veces, el LD es estimado a partir de blancos reactivos y un estándar, o por la desviación estándar del intercepto de una curva de calibración de blancos reactivos y estándares. En estos casos lo que se evalúa es el límite de detección instrumental y no el del método. Tomando en cuenta el riesgo $\alpha=0.05$ de la distribución normal del blanco y el riesgo $\beta=0.05$ de la distribución normal correspondiente al analito, la señal de detección (SD) se estima multiplicando la desviación estándar del blanco o la desviación estándar residual por 2 veces el valor de $z(=1.645)^{[15]}$ : SD=3.29 $s_{b}$. Si se utiliza la desviación estándar residual el LD se calcula dividiendo SD entre la pendiente de la curva de calibración por adición patrón.

El límite de cuantificación (LC) es la cantidad mínima que se puede cuantificar a un nivel de confiabilidad establecida. La señal de cuantificación (SC) es 10 veces la desviación estándar del blanco o la residual. Por tanto, el LC resulta de dividir SC entre el valor de la pendiente de la curva de calibración por adición patrón.

La robustez del método es la fortaleza que debe tener el método ante pequeños cambios en los factores involucrados en el proceso analítico en un mismo laboratorio ${ }^{[8]}$. Esto quiere decir que si se hacen pequeños cambios deliberados, de acuerdo a condiciones experimentales establecidas, los resultados no sufren variaciones significativas. Estos factores pueden ser el $\mathrm{pH}$, marca de reactivo, tipo de solvente, tipo de columna cromatográfica, analistas, etc.

Una vez evaluados los parámetros de validación, para la trazabilidad documental como evidencia objetiva, es necesario demostrar la validación a través de tres documentos: un procedimiento o protocolo de validación, el informe de validación y una declaración de la aptitud de que el método cumple con los requisitos del cliente $\mathrm{e}^{[4]}$.

Es importante señalar que existen muchas técnicas en las que no es aplicable la evaluación de todos los parámetros que se presentan en la figura 2. Por ejemplo, en volumetría clásica y en los métodos gravimétricos, la linealidad no tiene sentido. En la validación del método para determinar $\mathrm{pH}$ por potenciometría, los límites de detección y de cuantificación no son aplicables. En los métodos cualitativos los parámetros a validar son: límite de detección, falsos positivos, falsos negativos y la exactitud relativa (si aplica) $)^{[11]}$, robustez (si aplica). En algunos ensayos microbiológicos se evalúa la especificidad, la selectividad y el efecto de matriz ${ }^{[11]}$.

\section{NORMALIZACIÓN DE UN MÉTODO}

Una vez que el método ha sido validado se procede a elaborar el procedimiento normalizado como análisis de rutina. Un procedimiento normalizado es el protocolo establecido por el laboratorio, optimizado, validado, revisado, aprobado por la(s) persona(s) autorizada(s). Tiene una identificación única. Deben especificarse los parámetros que caracterizan el desempeño del método para la verificación.

Si el método ha sido desarrollado en el laboratorio es necesario optimizar y luego validar. La figura 3 presenta un flujograma de un método desarrollado en el laboratorio y su validación ${ }^{[16]}$, donde se pueden ver los dos procesos, el desarrollo (optimizado) y la validación. La optimización se puede llevar a cabo a través de dos vías, empírica y a través de un modelo matemático. La vía empírica se realiza a través de prueba y error y puede durar hasta 2 o más años en el desarrollo de 


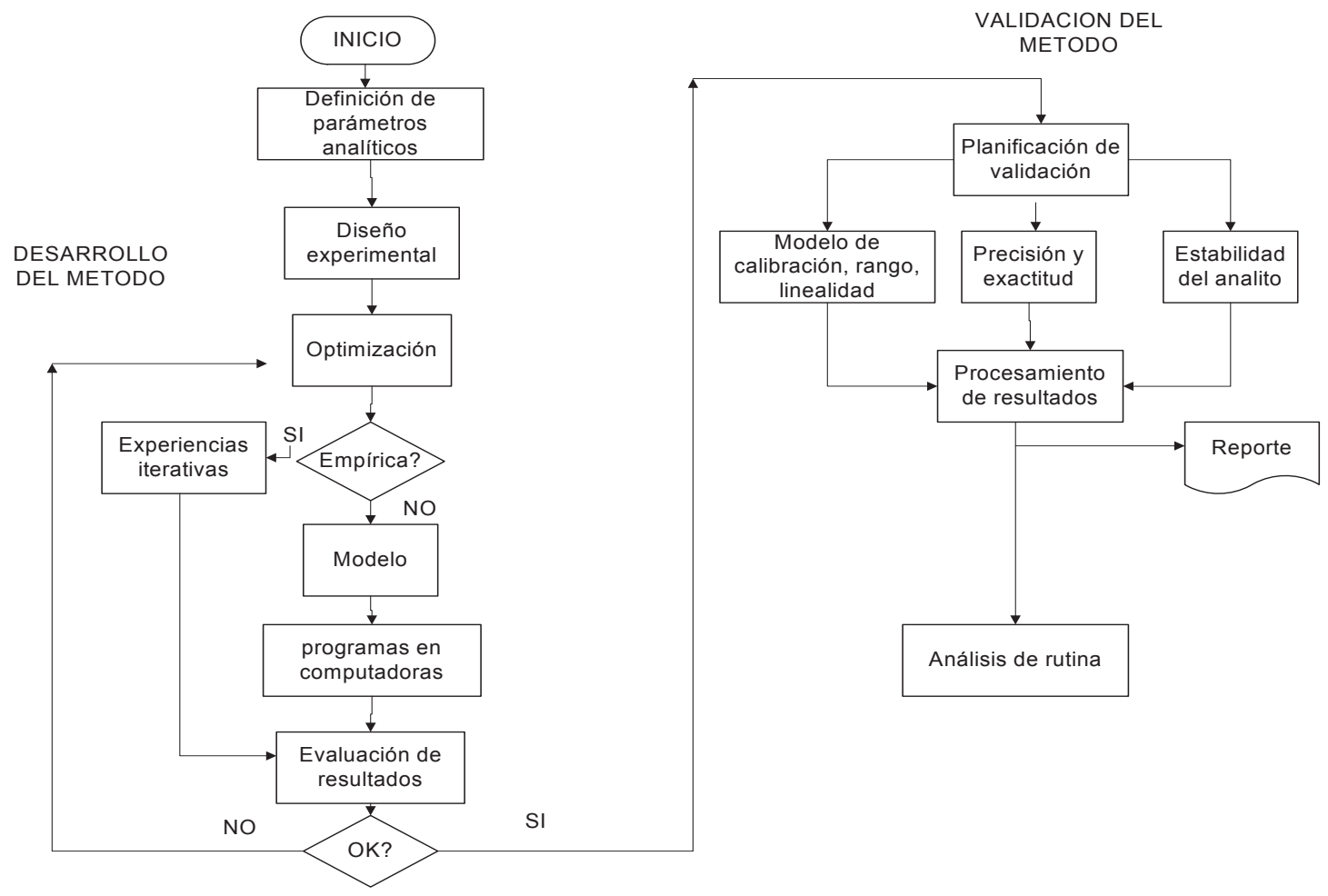

Figura 3. Desarrollo de un método y su validación.

un método. Mientras que, si se diseña el experimento utilizando un diseño experimental apropiado, se pueden desarrollar métodos en mucho menos tiempo. Muchas veces una optimización por el método empírico dura hasta 3 años y con un diseño experimental apropiado puede optimizarse solamente en 3 o 6 meses.

Muchos métodos en el campo de los alimentos, farmacéuticos, ambientales se encuentran en los manuales de métodos normalizados tales como: AOAC (Asociación Oficial de Químicos Analíticos de los Estados Unidos), USDA ( Departamento de Agricultura de los Estados Unidos), FDA (Administración de Drogas y Alimentos), EPA (Agencia de Protección del Medio Ambiente), AOCS (Asociación Americana de Químicos del Aceite), AACC (Asociación Americana de Química Clínica), ISO (Organización Internacional para la Normalización), USP (Farmacopea de los Estados Unidos), ASTM (Asociación Americana de Materiales y Ensayos), APHA (Asociación de Salud Pública Americana, métodos normalizados para análisis de agua).

Todos estos métodos son normalizados y en su contenido presentan los datos de la validación; por ejemplo, presentan los resultados de repetibilidad, reproducibilidad, bias (sesgo) o desviación del método, linealidad y rango, límite de detección y de cuantificación.
Estos datos de validación constituyen las especificaciones del método. En todos estos métodos se presenta implícitamente la declaración de la aptitud para el uso previsto. Es decir, la aplicabilidad en el tipo de matriz de la muestra y las condiciones que debe reunir el método para satisfacer los requisitos del cliente.

Dependiendo de los requerimientos y del propósito del método, se pueden clasificar según sea apto para cuantificar, identificar o detectar el analito en la muestra. La AOAC ${ }^{[11]}$ los clasifica en 6 categorías: 1. Confirmación de la identificación o detección del analito en la muestra; 2. Cuantificación a bajas concentraciones del analito; 3. Determinación del analito cuando está por encima o por debajo del límite de la concentración más baja, próximo al límite de cuantificación. 4. Cuantificación a altas concentraciones; 5. Determinación del analito cuando está presente por encima o por debajo del límite especificado de la concentración más alta; 6 . Ensayos cualitativos.

La ICH (Comisión Internacional de Armonización para el registro de requerimientos técnicos de fármacos de uso humano) clasifica los métodos a ser validados ${ }^{[17]}$ en: pruebas de identificación, pruebas cuantitativas para contenido de impurezas, pruebas de límite para el control de impurezas y pruebas cuantitativas para principios activos. 
Pero independientemente de la clasificación, la cantidad de parámetros a evaluar en la validación se seleccionará según la naturaleza del método, sea cuantitativo o cualitativo.

El contenido de algunos métodos normalizados de manuales se presentan en la tabla 1. Todos ellos son equivalentes. Cada uno de ellos tiene datos de validación. El alcance y los datos de validación constituyen las especificaciones del método. Esto representa los requisitos que reúne el método para el uso previsto.

\section{LA VERIFICACIÓN}

Se define como la aportación de evidencias objetivas de que un elemento satisface los requisitos especificados [7]. Si se aplica al método de ensayo se puede definir como: la confirmación a través de evidencias objetivas, que el método cumple con los requerimientos especificados para el uso previsto.

Si un laboratorio adopta un método normalizado, sin ninguna modificación, debe verificarlo antes de ser utilizado para los análisis de las muestras del cliente. Esto quiere decir que la verificación se limita a confirmar los datos de validación y el alcance del método para el uso que se tiene previsto para garantizar la calidad de los resultados al cliente.
En la verificación no se necesita la evaluación rigurosa $^{[12]}$ de los parámetros de desempeño del método. El laboratorio solamente necesita conocer las especificaciones del método normalizado a partir de los datos de validación que se reportan, tener analistas competentes, instrumentación calibrada, el medio ambiente e instalaciones apropiados, y los materiales de referencia y reactivos necesarios para efectuar el proceso de verificación.

Por ejemplo, para verificar la repetibilidad ${ }^{[11,18]}$ establecida en un método $s_{r M}$, se realiza la repetibilidad en el laboratorio (al menos 5 réplicas), se calcula la desviación estándar $s_{r}$ y se comparan las dos desviaciones estándares utilizando el test de Fisher. Este proceso puede aplicarse a tres niveles de concentración bajo, medio y alto.

Otra manera de verificar más rápidamente la repetibilidad es utilizando el límite de repetibilidad ${ }^{[19]}$, la cual establece que la diferencia máxima, en valor absoluto, entre dos replicas realizadas en condiciones de repetibilidad $\left(r_{p}\right)$ para un nivel de significancia seleccionado $(p=0.9545)$ debe cumplir la siguiente condición $r_{0.945}<2.83 s_{r M}$. De igual manera se puede aplicar para el límite de reproducibilidad, tal que las dos mediciones deben hacerse en condiciones de reproducibilidad.

Tabla 1. Contenido de algunos métodos normalizados.

\begin{tabular}{|c|c|c|c|}
\hline ISO & ASTM & APHA & AOAC \\
\hline $\begin{array}{ll}\text { - } & \text { Alcance (tipo de } \\
& \text { matriz e intervalo de } \\
& \text { concentraciones) } \\
\text { - } & \text { Referencias } \\
\text { normativas } \\
\text { - Principio } \\
\text { (fundamento del } \\
\text { método) } \\
\text { - Reactivos } \\
\text { (seguridad en } \\
\text { manejo cuando } \\
\text { aplica) } \\
\text { Aparatos (equipos y } \\
\text { cristalería) } \\
\text { Muestreo y } \\
\text { preparación de } \\
\text { muestras } \\
\text { Procedimiento } \\
\text { - Expresión de } \\
\text { resultados (modelo } \\
\text { matemático) } \\
\text { Interferencias } \\
\text { Precisión y exactitud }\end{array}$ & $\begin{array}{ll}\text { - } & \text { Alcance } \\
\text { - } & \text { Documentos } \\
\text { - } & \text { normativos } \\
\text { - } & \text { Terminología } \\
& \text { Resumen del } \\
& \text { método de ensayo } \\
\text { (principio) } \\
\text { - } & \text { Significado y uso } \\
& \text { (aptitud del método) } \\
\text { - } & \text { Aparatos } \\
\text { - } & \text { Procedimiento } \\
\text { - } & \text { Reporte (Cálculo y } \\
& \text { unidades) } \\
\text { - } & \text { Precisión y bias } \\
\text { - } & \text { Palabras claves } \\
\text { - } & \text { Anexos (si aplica) }\end{array}$ & 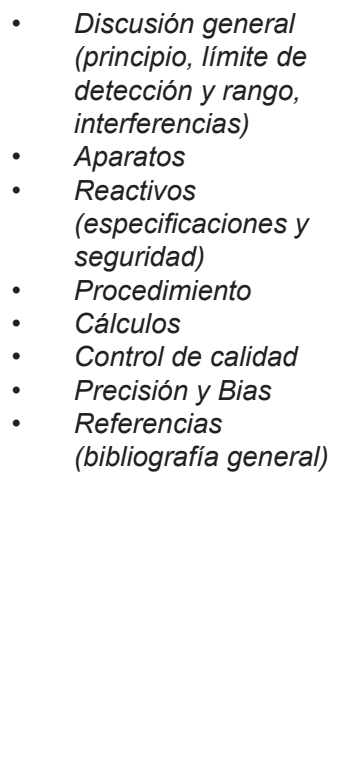 & $\begin{array}{ll}\text { - } & \text { Principio } \\
\text { - } & \text { (fundamento, } \\
\text { alcance y aptitud del } \\
\text { método, precisión } \\
\text { y bias) } \\
\text { - } \quad \text { Aparatos } \\
\text { Reactivos } \\
\text { (especificaciones, } \\
\text { seguridad) } \\
\text { Determinación } \\
\text { (precauciones, } \\
\text { directrices, cálculos) }\end{array}$ \\
\hline
\end{tabular}


Para verificar la exactitud se evalúa el sesgo[11,20] o desviación del método, se utilizan materiales de referencia, ya sea muestras de referencias certificadas o muestras de referencias internas. Se realizan $n$ veces, en condiciones de repetibilidad, se calculan el promedio $\bar{x}$ y la desviación estándar de las mediciones $s_{L}$ y se establece que la desviación del método debe cumplir la siguiente condición:

$$
|\bar{x}-\mu| \leq 2 \sqrt{s_{R}^{2}+s_{r}^{2}+\frac{s_{L}^{2}}{n}}
$$

Donde $\mu$ es el valor de referencia, $s_{R}$ y $s_{r}$ son los valores de desviación estándar de repetibilidad y reproducibilidad establecidos en la validación del método.

Si el método presenta desviaciones significativas, se debe realizar el cálculo de recobrado y efectuar las correcciones a los resultados de los análisis en las muestras. Cuando estas desviaciones existen, se le debe informar al cliente. La exactitud puede probarse a tres niveles de concentración, bajo, medio y alto.

Si el método normalizado establece un rango de linealidad, es decir, el intervalo de concentraciones donde la señal instrumental es proporcional a la concentración, lo que puede hacerse es verificar la linealidad a tres niveles de concentraciones (en los extremos y el centro del intervalo), efectuando al menos 3 lecturas en cada concentración. Los valores de 3 lecturas y 3 puntos se toman a partir de los requisitos de la estadística. El laboratorio puede adoptar otro diseño para evaluar la linealidad, todo es que sea estadísticamente válido.

Para verificar el límite de detección (LD) o de cuantificación (LC), se deben realizar corridas de muestras que tengan concentraciones próximas a los límites anteriores. En los ensayos cualitativos la verificación se limita a la especificidad y la precisión ${ }^{[11]}$.

La verificación de la calibración instrumental, es un aspecto muy importante a realizar antes de la verificación o validación del método. Cada instrumento es calibrado por personal competente en un laboratorio nacional de metrología o por personal especialista del proveedor o fabricante. En cualquiera de los casos, se extiende un certificado de calibración con las especificaciones técnicas correspondientes. El laboratorio deberá verificar el estado de calibración con sus patrones de referencia internos trazables a patrones nacionales o a materiales de referencia certificados.

Es importante señalar, que al igual que en la validación, se deben documentar los resultados de la verificación.
El laboratorio debe tener las evidencias objetivas de la verificación tales como: un procedimiento de verificación, un informe de los resultados de la verificación y una declaración que el método normalizado cumple con las especificaciones establecidas.

\section{DIFERENCIA ENTRE VALIDACIÓN Y VERIFICACIÓN}

$\mathrm{Si}$ se analizan los conceptos se observan las diferencias. La validación es la evaluación rigurosa de los parámetros de validación de un método que está en proceso de normalización. Una vez que se ha validado se elabora el procedimiento normalizado, especificando los parámetros de la validación. Mientras que, en la verificación se evalúan los parámetros que están especificado en el método normalizado, para observar si éste cumple con los requisitos en el medioambiente de trabajo.

Es decir que el usuario del método normalizado verifica que el desempeño del método cumple con los requisitos para los cuales fue validado. Las pruebas de verificación se realizan con pocos datos. Por ejemplo, en un diseño para evaluar la precisión en el proceso de validación (repetibilidad y precisión intermedia), se realizan 10 réplicas por día durante varios días (de 3 a 10 días) para reportar la desviación estándar de repetibildidad y de reproducibilidad interna o precisión intermedia. Para verificar esta precisión, puede ser suficiente realizar al menos 2 réplicas para verificar que cumple con lo especificado en el método normalizado.

\section{CONCLUSIÓN}

En la validación se determinan todos los parámetros posibles que caracterizan el desempeño del método, estableciendo las especificaciones para el uso requerido por los clientes. En la verificación se confirman estas especificaciones en el medio ambiente de trabajo. Para verificar un método normalizado no se necesitan evaluaciones rigurosas.

Los laboratorios no necesitan validar si utiliza métodos normalizados. Solamente verifican y los documentan para las evidencias objetivas en el proceso de acreditación. Los auditores deben tener clara esta diferencia. Pues es muy frecuente observar la confusión entre validación y verificación cuando se realizan las evaluaciones in situ. En algunas normas nacionales sustituyen el término verificación por confirmación, teniendo el mismo significado. Es importante puntualizar que si estos requisitos no se cumplen en un laboratorio de ensayo no puede obtener la acreditación. 


\section{AGRADECIMIENTO}

Un reconocimiento a la Lic. Adela Miranda, responsable de la Oficina Nacional de Acreditación (ONA) del Ministerio de Fomento Industria y Comercio (MIFIC), por haber sugerido el tema, el cual fue expuesto y discutido ante los auditores de la ONA, en Managua. Un reconocimiento también a Erick Méndez, de la ONA, por su valioso aporte en las definiciones de validación y verificación a partir de la VIM 2008. Igualmente el autor hace extensivo este reconocimiento a la Lic. Gilma Molina, de la Oficina Salvadoreña de Acreditación del CONACYT en El Salvador, por sus inquietudes de homologar los criterios de evaluación a nivel centroamericano.

\section{REFERENCIAS BIBLIOGRÁFICAS}

1. GATT, (1994), Ronda de Uruguay sobre obstáculos técnicos al comercio. En internet: http://www.SICE OMC - GATT Acta Final de la Ronda Uruguay.mht. Acceso el 19/10/09.

2. LA GACETA, (1996), Diario Oficial del Gobierno de Nicaragua. Ley 219.

3. ONA-MIFIC, Oficina Nacional de Acreditación. En internet: http://www.mific.gob.ni/dirMercados/ concredito.htm. Acceso el 16/10/09.

4. ISO/IEC 17025(ES) (2005). "Requisitos Generales para la Competencia de los Laboratorios de Ensayo y de Calibración", Norma Internacional, Ginebra, Segunda Edición.

5. ISO/IEC 15189 (E) (2007). "Requisitos particulares para la Calidad y Competencia Técnica de los Laboratorios Clínicos", Norma Internacional, Ginebra, Segunda Edición.

6. ISO/IEC 17000:2004 (ES), (2004). "Evaluación de la conformidad. Principios y vocabulario", Norma Internacional, Ginebra.

7. a) VIM 200:2008, "Vocabulario Internacional de Metrología", Edición en Español, BIPM, París.. b) ISO 9000:2005 (ES), "Sistemas de Gestión de la Calidad. Conceptos y Vocabulario", Norma Internacional, Ginebra.

8. EURACHEM/CITAC, (1998), "fitness for purpose of analytical methods", UK.
9. ILAAC G9:2005, Guía para el uso de materiales de referencia. Acceso en Internet: http://www. organismouruguayodeacreditacion.org/IAAC.

10. FINGBERG M., (1998), "Validation de méthodes", Editorial Masson, Paris. Pag.56

11. ALACC (Criterios del Comité de Acreditación de Laboratorios Analíticos), (2007), "Requirements for Methods Verifications", AOAC International.

12. NATA/TN (National Association of Testing Authorities, Australia), (2009), "Guidelines for the validation and verification of test methods".

13. FUNK W., DANMAMN V. y DONNVERT G, (1995), "Quality Assurance in Analytical Chemistry", VCH, Alemanis, pag. 28.

14. MILLER J.N., MILLER J.C., (2002), "Estadística y quimiometría para química analítica", Pearson Educación S.A., Madrid, pag. 127.

15. RAMIS G. y ALVAREZ-COQUE M.A., (2001), "Quimiometria", Editorial Síntesis, Madrid, pág. 124.

16. DOWALL R. D., (1999), "The role of laboratory information management systems (LIMS) in analytical method validation", Analitica Chimica Acta, 391, 149-158.

17. ICH Q2(R1), (2005), "Validación de procedimientos analíticos".

18. ISO/TS 21748:2004, "Guidelines for the use of repeatibility, reproducibility and trueness estimates in measurement uncertainty estimation". Documento de Especificación técnica.

19. ISO 5725-1 (1994), "Accuracy of measurement methods and results".

20. NEUILLY M., (1998), "Controle de performances des measures industrielles", Tec- Doc, Paris, pag. 41. 